\title{
Analysis of Thin Films by Time-of-Flight Low Energy Ion Scattering
}

\author{
S. PrØša*, M. Kolíbal, P. BÁBor, J. Mach And T. ŠIKOla \\ Institute of Physical Engineering, Brno University of Technology \\ Technicka 2896/2, 61669 Brno, Czech Republic
}

(Received September 1, 2006; revised version March 5, 2007; in final form April 10, 2007)

\begin{abstract}
In the paper the design and application of a time-of-flight low energy ion scattering instrument built into an UHV complex deposition and analytical apparatus is described. A special attention is aimed at demonstrating the ability of time-of-flight low energy ion scattering to analyse near-to-surface layers of thin films prepared both ex situ and in situ. It is shown that the broadening of peaks in time-of-flight low energy ion scattering spectra can be attributed to multiple scattering and inelastic losses of ions in deeper layers. As a result of that, the peak width of ultrathin films depends on their thickness.
\end{abstract}

PACS numbers: 68.49.Sf, 81.05.Cy, 68.55.-a, 81.15.Ef

\section{Introduction}

Low energy ion scattering (LEIS), alternatively called ion scattering spectroscopy (ISS), belongs to a group of surface analytical techniques with a unique surface sensitivity. It is generally accepted that LEIS is capable of sensing the outermost surface monolayer [1]. This is particularly true if electrostatic energy analysers are applied for measuring energy of scattered ions [2]. However, in the case of the time-of-flight (TOF) detection technique, not only scattered ions but also neutral particles are detected [3]. These particles are formed by neutralization of ions scattered mostly at deeper layers of a solid surface $[4,5]$. Hence, the TOF-LEIS possesses information on deeper layers as well and this technique can be used for an analysis of ultrathin films and in situ monitoring of their growth. The paper shows examples of such applications. A special attention is paid to a qualitative explanation of the obtained spectra and shapes of the peaks.

*corresponding author; e-mail: prusa@fme.vutbr.cz 


\section{Experimental}

The TOF-LEIS spectrometer is built into a UHV chamber (see Fig. 1), which is a part of a complex deposition and analytical UHV apparatus equipped with additional analytical techniques such as secondary ion mass spectroscopy (SIMS), X-ray photoelectron spectroscopy (XPS), Auger electron spectroscopy (AES), low-energy electron diffraction (LEED), thermal desorption spectroscopy (TDS), and ellipsometry. The base pressure in the chamber is $4 \times 10^{-9}$ mbar or lower.

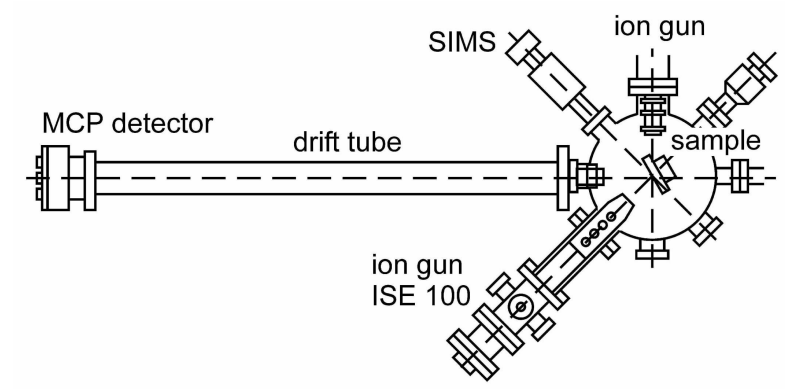

Fig. 1. Schematic of a TOF-LEIS experimental setup.

The experimental setup consists of an ion source, a sample manipulator, and a drift tube. At the end of the drift tube a three-stage microchannel plate (MCP) detector is placed. A differentially pumped ion source (OMICRON ISE 100) produces gas ion beams (e.g. $\mathrm{He}^{+}, \mathrm{Ne}^{+}$, and $\mathrm{Ar}^{+}$) in the energy range from $500 \mathrm{eV}$ to $5 \mathrm{keV}$. After acceleration the ion beam is chopped over a removable screen-plate slit and so ion pulses of a time width $\geq 110 \mathrm{~ns}$ (full-width at half maximum - FWHM) are produced. The size of the ion beam spot at a sample area is set to $2-3 \mathrm{~mm}$ in diameter. All analyses were performed using an ion dose below $10^{11}$ ions $/ \mathrm{cm}^{2}$ to ensure a negligible damage induced by ions at the surface. The sample manipulator provides an $x-y-z$ linear motion, as well as a rotation along the $z$-axis of the manipulator (polar angle), and an azimuthal rotation around the axis perpendicular to the sample plane. The incidence angle was kept at $20^{\circ}$ with respect to the sample normal and particles were detected at a fixed scattering angle of $135^{\circ}$ within a detector entrance angle of $2^{\circ}$. The path length of the scattered particles was $110 \mathrm{~cm}$ (can be extended to $205 \mathrm{~cm}$ ).

A sample can be thermally treated (degassing, annealing, flashing) by thermal radiation, electron bombardment, or by electric current going through the sample. To clean the sample, ion beam sputtering by the ion source specified above can be carried out as well.

Having been scattered, a fraction of the particles enters the drift tube. To prevent collecting the particles additionally scattered from the walls of the chamber or the drift tube, diaphragms have been installed into the drift tube to stop them. 
At the end of the drift tube, the scattered particles are detected by the MCP detector. Both ionized and neutral particles are detected.

The measured time of flight of scattered particles is a time interval between a trigger pulse (the formation of an ion packet by chopping the beam) and an individual signal pulse (a scattered particle hits the MCP detector). The data are finally recorded and processed by an electronics (Ortec) consisting of a time-toamplitude convertor (TAC) and a multichannel pulse analyser card.

The work pressure in the chamber during the experiment was kept in a range of $10^{-8}$ mbar. An e-beam effusion cell (Omicron) mounted to the chamber (Fig. 1) was used for deposition experiments (gallium ultrathin layers). Evaporation rates of the cell were calibrated by a crystal quartz monitor. A plasma ion beam source, developed in the group, serves as a tool for direct deposition of ultrathin films (ultralow energy mode $E \leq 100 \mathrm{eV}$ ) and modification of surfaces. Both the ion gun and e-beam effusion cell can be simultaneously used for the deposition of ultrathin layers of gallium nitride.

\section{Results and discussion}

The thin film deposition and modification by very low energy ion beams needs to be in situ monitored. The described TOF-LEIS spectrometer was designed to give fast quantitative information during the deposition processes rather than high resolution results. Additionally, in some cases TOF-LEIS may also give reasonable information on scattering processes in thin films prepared externally.

The position of the peaks in LEIS spectra can be easily calculated from the binary collision model (BCM) [2]. However, this model is not sufficient when all the spectra features need to be explained. Multiple scattering, structural effects (shadowing, blocking), scattering cross-section, non-elastic energy losses, and experimental parameters, all of them leave their own foot-print in the spectra. Generally, it is difficult to analyse precisely all of their impacts in LEIS spectra at the same time and it goes out of the scope of our article. But some qualitative information can be obtained when a certain surface is analysed under different experimental conditions.

A comparison of three TOF-LEIS spectra for helium, neon, and argon scattering on an ex situ prepared gold-silicon system can be seen in Fig. 2. The spectra were normalized to the same height. The gold film (30 nm thick) was deposited on $\mathrm{Si}(111)$ substrate (covered with native oxides) in a Polaron E5170 apparatus by magnetron sputtering technique from a high purity gold target. After the deposition the sample was exposed to ambient atmosphere during the transfer into the UHV apparatus.

The sample surface was first cleaned by $\mathrm{Ar}^{+}$ion sputtering in situ and then analysed by TOF-LEIS. The first spectrum with a peak at $2.5 \mu$ s represents the scattering of $4.5 \mathrm{keV} \mathrm{He}{ }^{+}$ions from this surface. The relatively large peak width $(\mathrm{FWHM}=0.95 \mu \mathrm{s})$ is caused by multiple scattering and inelastic losses (some of 


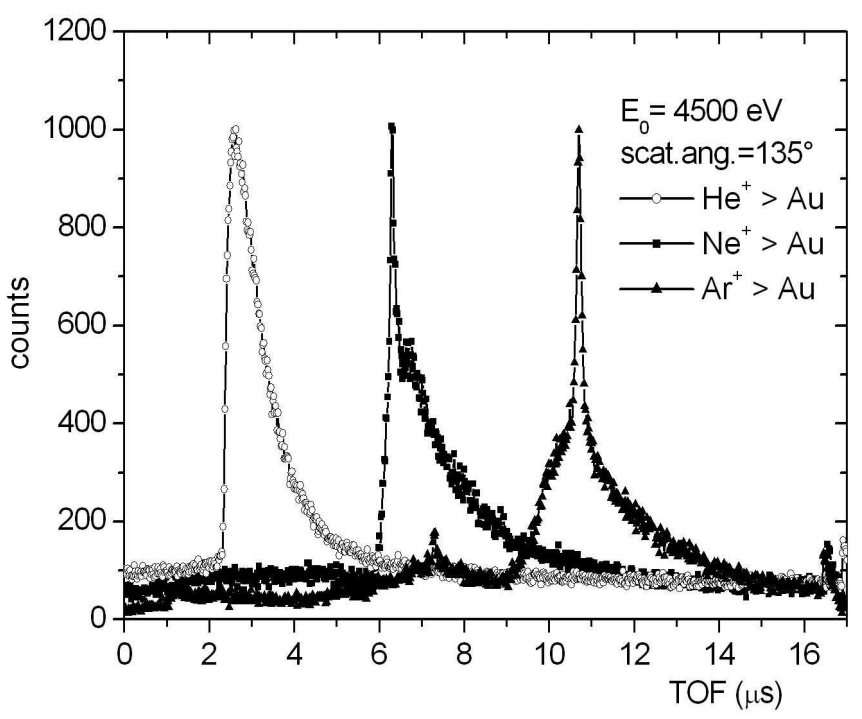

Fig. 2. Three spectra representing the scattering of helium, neon, and argon ions from the gold thin film (30 nm thick) deposited on silicon substrate.

these effects are described at [6] for polycrystalline copper sample) in subsurface regions of a relatively thick gold film. The spectrum on the right was taken by the scattering of $\mathrm{Ar}^{+}$ions with an energy of $4.5 \mathrm{keV}$ from the same sample. At $10.7 \mu \mathrm{s}$ a sharp maximum is present and it corresponds to a single scattering of ions from near-to-surface gold layers. As the interaction of heavy argon ions with gold atoms is more intensive than in the case of helium (higher scattering cross-section), the penetration of argon ions into deeper layers is less effective, and thus the single ion backscattering on gold atoms of the outermost layers becomes significant. Both the tail of the peak and broadening in the front part of the peak result from the effects of multiple scattering events $[2,5]$ since both scattered ions and neutrals are detected. A small peak at $7.4 \mu \mathrm{s}$ has emerged in the spectra due to the scattering of double ionized argon ions.

Light helium ions penetrate deeper under the gold surface than the argon ions and undergo more multiple scattering events. As a result of that, and consequently of higher inelastic losses due to longer paths, the corresponding peak of the backscattered helium ions is broader as it can be seen from the first spectrum of Fig. 2. The last spectrum (in the middle) has a sharp onset at $6.3 \mu$ s and corresponds to the scattering of neon ions $(4.5 \mathrm{keV})$ from the same sample. The peak is $0.75 \mu$ s wide (FWHM) and represents a transition case between the spectra on the left and right which results from the relation of neon mass towards helium and argon.

The gold film was deposited on $p$-doped Si(111) substrate covered with the native oxide. A peak attributed to the scattering from silicon atoms is not visible 
in any spectrum. In principle argon cannot be scattered from silicon or oxygen under a scattering angle of $135^{\circ}$ (only forward scattering of heavier ions from lighter target atoms is possible). Backscattering of neon from silicon is in principle possible. However, during this scattering the incident neon ions would lose $97 \%$ of their primary energy and due to the small detection efficiency of the MCP detector to the ions or atoms of low energies $(<500 \mathrm{eV})[7]$ the backscattered neon could not be detected. Hence, the only real possibility to analyse silicon atoms remains to apply helium ions. The silicon peak can be observed in Fig. 3 and it will be described later. However, no such a peak appears in the spectra taken on a gold layer. The gold is known to form islands on silicon substrates for lower coverage, but in our case the gold film was $30 \mathrm{~nm}$ thick, which is enough to completely cover the substrate and to shield the silicon atoms from the incident ion beam.

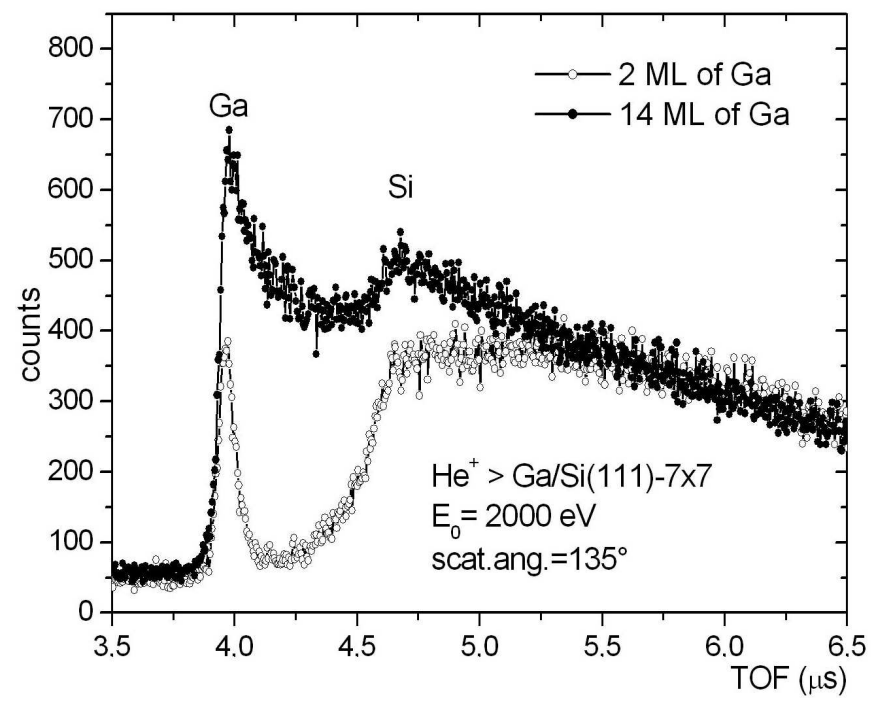

Fig. 3. TOF-LEIS spectra of gallium films deposited on $\mathrm{Si}(111)-7 \times 7$ substrate (incident helium ions, $2000 \mathrm{eV}$ ). The spectra represent scattering from the gallium films with a thickness of $2 \mathrm{ML}$ and $14 \mathrm{ML}$, respectively.

When using surface sensitive methods like LEIS, it is necessary to reduce surface contamination. Since the energy loss of $\mathrm{He}^{+}$projectiles scattered on such light adsorbates (e.g. carbon, oxygen) is much higher than if they are scattered on gold atoms they contribute to the tail of the surface peak. However, in the spectra in Fig. 2 these peaks are overshadowed by the peak broadening due to the effect of multiple scattering in the gold film.

Since TOF-LEIS is in practice a non-destructive method, it is a proper tool for in situ analysis of ultrathin film growth. An $\mathrm{Si}(111)$ sample with a $7 \times 7$ reconstruction was used as a substrate for gallium-growth experiments. The substrate was cut from the standard silicon wafer. After each partial one monolayer 
$\left(1 \mathrm{ML}=7.84 \times 10^{14}\right.$ atoms $\left./ \mathrm{cm}^{2}\right)$ deposition carried out by the e-beam effusion cell, the sample was rotated towards the spectrometer and analysed in situ. Two of the spectra obtained by the scattering of $2 \mathrm{keV} \mathrm{He}^{+}$ions from $2 \mathrm{ML}$ and $14 \mathrm{ML}$ thick gallium film are demonstrated in Fig. 3. A sharp narrow peak related to the single scattering on gallium atoms appears at $3.9 \mu \mathrm{s}$ in the spectrum for 2 monolayers of deposited gallium. The peak is followed by a plateau with an onset at $4.6 \mu \mathrm{s}$. The plateau is a main part of the very wide silicon peak with the slowly decreasing tail intensity. The silicon substrate is almost infinitely thick in comparison with the deposited films of gallium and gold described in this article. The silicon peak width is increased by the multiple scattering and the inelastic energy losses in deeper subsurface regions. In the second spectrum representing the scattering from $14 \mathrm{ML}$ thick gallium film the single and multiple scattering on gallium atoms is dominant, while the scattering on silicon atoms is suppressed (increased area of gallium islands) and overlapped by the gallium peak tail. A small peak at $4.6 \mu \mathrm{s}$ indicates residual scattering on silicon atoms. The gallium film grows on the $\mathrm{Si}(111)-7 \times 7$ substrate in these experimental conditions in an island growth mode [8] and the substrate atoms are not completely screened off by gallium atoms.

The inelastic energy losses and the multiple scattering also influence the width and shape of the gallium peak as can be seen from the peak comparison in Fig. 3. The dependence of these peak characteristics on the layer thickness is discussed in [8] and [9]. In this way, TOF-LEIS can yield some information on the growth mode and morphology of ultrathin films.

\section{Conclusions}

In the paper the design and application of the TOF-LEIS instrument built into an UHV complex deposition and analytical apparatus was presented. A special attention was aimed at demonstrating the ability of TOF-LEIS to analyse near-to-surface layers of thin films prepared both ex situ and in situ. It was stated that the broadening of the peaks can be attributed to multiple scattering of ions in deeper layers and to their inelastic energy losses. As a result of that, the peak width of ultrathin films depends on their thickness.

Taking into account the fact that the scattered non-neutralised ions, which mostly undergo collisions with atoms from the outermost layer, can be separated from neutrals in time and, hence, detected by the TOF technique, the unique surface sensitivity of LEIS remains also valid for TOF-LEIS. In this way, TOF-LEIS is capable of detecting a quality of both outermost and deeper layers (e.g. layer continuity, growth modes, pinholes) becoming thus a proper tool for the investigation of ultrathin films, for instance increasingly demanded high- $k$ dielectric films. 


\section{Acknowledgments}

This work was supported by the research grant programmes of the Ministry of Education CR (projects No. MSM0021630508, and LC1360011) and GAAV (project No. IAA1010413).

\section{References}

[1] H.H. Brongersma, P.M. Mul, Chem. Phys. Lett. 14, 389 (1972).

[2] J.M. Walls, Methods of Surface Analysis, Cambridge University Press, Cambridge 1988, p. 264, 270.

[3] M. Draxler, S.N. Markin, S.N. Ermolov, K. Schmid, C. Hesch, A. Poschacher, R. Gruber, M. Bergsmann, P. Bauer, Vacuum 73, 39 (2004).

[4] D.P. Woodruff, T.A. Delchar, Modern Techniques of Surface Science, Cambridge University Press, Cambridge 1992, p. 195.

[5] H. Niehus, W. Heiland, E. Taglauer, Surf. Sci. Rep. 17, 213 (1993).

[6] M. Draxler, S.M. Markin, M. Kolíbal, S. Pr ůša, T. Šikola, P. Bauer, Nucl. Instrum. Methods Phys. Res. B 230, 398 (2005).

[7] Hamamatsu MCP Assembly, Technical Information, Cat. No. TMCP9001E03, Hamamatsu Photonics K.K., 1991.

[8] M. Kolíbal, S. Průša, M. Plojhar, P. Bábor, T. Šikola, Nucl. Instrum. Methods Phys. Res. B 249, 318 (2006).

[9] M. Kolíbal, S. Pr ůša, P. Bábor, T. Šikola, Surf. Sci. 566-568, 885 (2004). 